\title{
Comparative analysis of the molecular mechanism of resistance to vapendavir across a panel of picornavirus species
}

\author{
Kristina Lanko ${ }^{\mathrm{a}, 1}$, Liang Sun ${ }^{\mathrm{a}}$, Mathy Froeyen ${ }^{\mathrm{b}}$, Pieter Leyssen ${ }^{\mathrm{a}}$, Leen Delang ${ }^{\mathrm{a}}$, \\ Carmen Mirabelli $^{\mathrm{c}}$, Johan Neyts ${ }^{\mathrm{a}, *}$ \\ ${ }^{a}$ KU Leuven - University of Leuven, Department of Microbiology and Immunology, Rega Institute for Medical Research, Laboratory of Virology and Chemotherapy, B- \\ 3000, Leuven, Belgium \\ ${ }^{\mathrm{b}}$ KU Leuven - University of Leuven, Department of Pharmaceutical and Pharmacological Sciences, Rega Institute for Medical Research, Laboratory of Medicinal \\ Chemistry, B-3000, Leuven, Belgium \\ ${ }^{\mathrm{c}}$ University of Michigan Medical School, Ann Arbor, MI, USA
}

\section{A R T I C L E I N F O}

\section{Keywords:}

Antivirals

Enterovirus

Drug resistance

Capsid binders

\begin{abstract}
A B S T R A C T
Vapendavir is a rhino/enterovirus inhibitor that targets a hydrophobic pocket in the viral capsid preventing the virus from entering the cell. We set out to study and compare the molecular mechanisms of resistance to vapendavir among clinically relevant Picornavirus species. To this end in vitro resistance selection of drugresistant isolates was applied in rhinovirus 2 and 14, enterovirus-D68 and Poliovirus 1 Sabin. Mutations in the drug-binding pocket in VP1 (C199R/Y in hRV14; I194F in PV1; M252L and A156T in EV-D68), typical for this class of compounds, were identified.

Interestingly, we also observed mutations located outside the pocket (K167E in EV-D68 and G149C in hRV2) that contribute to the resistant phenotype. Remarkably, the G149C substitution rendered the replication of human rhinovirus 2 dependent on the presence of vapendavir. Our data suggest that the binding of vapendavir to the capsid of the G149C isolate may be required to stabilize the viral particle and to allow efficient dissemination of the virus. We observed the dependency of the G149C isolate on other compounds of this class, suggesting that this phenotype is common for capsid binders. In addition the VP1 region containing the G149C substitution has not been associated with antiviral resistance before. Our results demonstrate that the phenotype and genotype of clinically relevant vapendavir-resistant picornavirus species is more complex than generally believed.
\end{abstract}

\section{Introduction}

The Picornaviridae family comprises numerous human pathogens, belonging to species Enterovirus A-D and Rhinoviruses A-C. Clinical manifestations of picornavirus infections range from mild (e.g. common cold caused by Rhinoviruses (hRV); hand-foot-and-mouth disease by coxsackievirus A (CVA), enterovirus 71 (EV-A71) and others) to lifethreatening (myocarditis, encephalitis, acute flaccid paralysis), although many infections remain subclinical with no apparent symptoms (Tapparel et al., 2013).

Human rhinoviruses are the major causative agents of common cold affecting millions of people worldwide (Passioti et al., 2014). Rhinoviruses have been shown to not only cause mild upper respiratory disease but also to provoke the exacerbations of preexisting respiratory conditions like COPD and asthma (Gern, 2015; Mallia et al., 2011). EV-D68 recently emerged as another picornavirus with respiratory tropism (Imamura and Oshitani, 2015). In addition, during recent outbreaks of EV-D68 the link between this viral infection and neurological complications (acute flaccid myelitis, AFM) has become clear (Aliabadi et al., 2016; Messacar et al, 2016, 2018; Mishra et al., 2019; Uprety et al., 2019). Despite the great success of the Global Polio Eradication Initiative (GPEI), poliovirus (PV) will remain a threat even after complete eradication of the wild-type viruses, e.g. due to circulation of vaccine-derived viruses or accidental virus release ("Polio Now - GPEI," n.d.). Development of antiviral drugs to control and prevent potential outbreaks is one of the priorities in the polio endgame (Collett et al.,

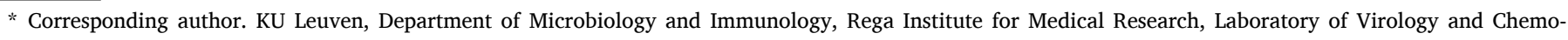
therapy, Herestraat 49 - box 1043, 3000, Leuven, Belgium.

E-mail address: johan.neyts@kuleuven.be (J. Neyts).

1 Present address: Kristina Lanko, Department of Clinical genetics, Erasmus medical center, Dr.Molewaterplein 40, 3015 GD, Rotterdam, The Netherlands.
} 
2016).

To date there is no specific antiviral therapy for any of the picornavirus infections. Vaccines are only available for poliovirus (live-attenuated oral polio vaccine and inactivated vaccine) (Kirkpatrick and Mychaleckyj, 2015) and EV-A71 (only approved in China) (Liang and Wang, 2014). Several classes of early stage inhibitors have been developed, of which some entered clinical trials (Collett et al., 2016; Hayden et al, 1995, 2003). The first class of antiviral compounds against picornaviruses that was discovered are the WIN-like capsid binders. The first WIN-molecules were discovered in 1977 and were active against rhinoviruses (Diana et al., 1977). Many analogs were synthesized and optimized to target specific enteroviruses. One of the first compounds, WIN 38020 (also known as arildone), exerted antiviral activity against poliovirus both in vitro and in vivo (McKinlay et al., 1982). WIN-like compounds such as pirodavir, pleconaril, pocapavir and vapendavir bind to the pocket located in the structural protein VP1 in the canyon - a structure present in most picornaviruses (Baggen et al., 2018). This region plays a major role in early stages of picornavirus infections (attachment, entry and uncoating). Capsid binders increase the stability of the viral capsid, thus preventing conformational changes necessary for receptor interaction and uncoating. Pleconaril, pirodavir (Hayden et al., 1995) and vapendavir reached Phase II clinical trials for anti-rhinovirus activity; pleconaril and vapendavir showed a beneficial effect against the infection (Hayden et al., 2003; Matz, 2013). The development of both pleconaril and pirodavir has been halted; however, pleconaril was also tested for other indications in infants with enterovirus meningitis (inconclusive results) (Abzug et al., 2003) and enterovirus sepsis (positive trend observed) (Abzug et al., 2015). Vapendavir is the only WIN-compound for which development is still ongoing with proven reduction of viral load in hRV infected adults in phase IIb clinical trials without significant effect on reduction of asthma exacerbations (Matz, 2013) (ClinicalTrials.gov: NCT02367313). Like other capsid binders, vapendavir exerts broad-spectrum activity against different enteroviruses including EV-A71, EV-D68, PV, and human rhinoviruses (Feil et al., 2012; Rhoden et al., 2013; Sun et al., 2015; Tijsma et al., 2014a). One of the drawbacks of capsid binders is the rapid development of resistance in vitro (Feil et al., 2012; Lacroix et al., 2014; Shepard et al., 1993; Sun et al., 2015) and in men (Collett et al., 2016; Hayden et al., 2003). Typical mutations that have been reported to be associated with resistance to capsid binders are I99F in hRV2, A150 T/V, C199R, V188I and E276K in hRV14; V69A and K155E in EV-D68; A24V and I194 F/M in PV1. The selection of vapendavir-resistant isolates has not been studied yet, however the cross-resistance of pleconaril-resistance rhinoviruses to vapendavir (Feil et al., 2012) suggests similar mechanisms of virus adaptation. Given the clinical potential of vapendavir as anti-rhinovirus compound and its broad-spectrum activity, it is of interest to reveal the resistance profile of enterovirus species, sensitive to vapendavir. Here, we explore the particular characteristics of resistance development of four enterovirus species (hRV14, hRV2, EV-D68 and PV) to vapendavir.

\section{Materials and methods}

\subsection{Cells and viruses}

All cell types used in this study [Buffalo green monkey (BGM) cells (ECACC 90092601), Human Caucasian embryo rhabdomyosarcoma (RD, ECACC 85111502), Epithelium cells cervix adenoncarcinoma (HeLa) kindly provided by Dr. K. Andries (Janssen Pharmaceutica, Belgium)] were cultured in MEM Rega3 medium (Gibco) supplemented with 10\% FBS, $2 \mathrm{mM}$ L-glutamine (Gibco) and $0.075 \%$ sodium bicarbonate (Gibco) at $37{ }^{\circ} \mathrm{C}$ in $5 \% \mathrm{CO}_{2}$ incubators.

Viral infections were carried out in the same medium supplemented with 2\% FBS. hRV and EV-D68 infections were carried out in the culture medium which was supplemented with $30 \mathrm{mM} \mathrm{Mg}^{2+}$ at $35{ }^{\circ} \mathrm{C}$ in $5 \% \mathrm{CO}_{2}$ incubators.
PV1 (Sabin) was derived from the infectious clone pT7/S1F, which was kindly provided by A.J. Macadam (McGoldrick et al., 1995) and virus stocks were produced in BGM cells. The EV-D68 strain CU70 was obtained from RIVM (Bilthoven, The Netherlands) and cultured in HeLa cells. hRV2 and hRV14 were kindly provided by Dr. K. Andries (Janssen Pharmaceutica, Belgium) and cultured in HeLa cells.

\subsection{Compounds}

Vapendavir (BTA798) was provided by Vaxart Inc., USA. Pirodavir was synthesized by Prof. G. Pürstinger (University of Innsbruck). Pleconaril was kindly provided by V. Makarov (RAS Institute of Biochemistry, Russia). Compound stocks were prepared in DMSO at a concentration of $10 \mathrm{mM}$ and diluted in cell culture medium for experimental use.

\subsection{5-Step resistance selection protocol}

The detailed description of the protocol has been published previously (Delang et al., 2014). In brief, optimal concentrations of vapendavir and virus input were determined for each virus. Optimal conditions are defined as the lowest compound concentration and highest virus input still resulting in complete inhibition of viral-induced CPE. Three identical 96-well plates were set up with the optimal condition and development of CPE was monitored microscopically. The supernatant from the wells exhibiting CPE (potentially resistant virus) was passaged in the presence of vapendavir to obtain a virus stock and the sensitivity of this stock was tested in an antiviral assay.

\subsection{Antiviral assays}

Cells were seeded in 96-well plates (HeLa 15*10 ${ }^{3}$ cells/well; BGM $20 * 10^{3}$ cells/well) in $2 \%$ FBS containing MEM Rega 3 medium and were kept overnight at $37{ }^{\circ} \mathrm{C}$ or $35{ }^{\circ} \mathrm{C}$ (for hRV) $5 \% \mathrm{CO}_{2}$ to allow attachment. The next day serial dilutions of the compounds were added to the cells. Cultures were infected with viruses at a MOI 0,01 and were kept until full CPE was observed in virus control ( $24 \mathrm{~h}$ for PV1-Sabin, 3 days for other viruses in this study). Microscopic evaluation and cell viability readout with MTS reagent [3-(4,5-dimethylthiazol-2-yl)-5-(3-carboxymethoxyphenol)-2-(4-sulfophenyl)-2H-tetrazolium, inner salt; Promega] were performed for assessing the activity of compounds.

\subsection{Replication kinetics}

HeLa cells were seeded in 48-well plates and infected with MOI 2 for $1 \mathrm{~h}$ at $35{ }^{\circ} \mathrm{C}$. After removal of the inoculum, cultures were washed 3 times with PBS and fresh medium was added. Intracellular RNA was isolated at indicated timepoints with the RNeasy mini kit (Qiagen) and quantified by qRT-PCR.

\subsection{Thermostability assay}

HRV2 WT or C3 isolate stocks were incubated at $37^{\circ} \mathrm{C}$ for $15 \mathrm{~min}$ and then $2 \mathrm{~min}$ at temperatures between 37 and $62{ }^{\circ} \mathrm{C}\left(5^{\circ} \mathrm{C}\right.$ increment $)$ in presence of $5 \mu \mathrm{M}$ vapendavir or DMSO solvent. The samples were cooled down to $4{ }^{\circ} \mathrm{C}$ and then the infectivity was determined by end-point titration.

\section{7. $q R T-P C R$}

qRT-PCR was performed using the BioRad iTaq Universal SYBR Green kit with pan-enterovirus primers ENRI 4- and ENRI 3+ (Lönnrot et al., 1999) on Applied Biosystems 7500 Fast Real-Time PCR System. A geneBlock (IDT) standard corresponding to the target region of the primers (5'UTR) was included for quantification of viral RNA copies. 
Table 1

Vapendavir-resistant isolates carry mutations in VP1 and are crossresistant to other capsid binders. Antiviral activity of capsid binders was assessed in a CPE-reduction assay. Data are mean values of 3 independent experiments $\pm S D$. NA - not active.

\begin{tabular}{|c|c|c|c|c|}
\hline \multirow[b]{2}{*}{ Virus } & \multirow[b]{2}{*}{$\begin{array}{l}\text { Genotype } \\
\text { VP1 }\end{array}$} & \multicolumn{3}{|c|}{$\mathrm{EC}_{50} \mu \mathrm{M}$ (fold change $\mathrm{EC}_{50}$ ) } \\
\hline & & Vapendavir & Pleconaril & Pirodavir \\
\hline \multirow[t]{3}{*}{ hRV14 } & WT & $0.09 \pm 0.01$ & $0.20 \pm 0.005$ & $0.14 \pm 0.1$ \\
\hline & C199R & $>10(>110)$ & $\begin{array}{l}2.9 \pm 1.0 \\
(14.5)\end{array}$ & $>27(>192)$ \\
\hline & C199Y & $>10(>110)$ & $3.4 \pm 1.6(17)$ & $\begin{array}{l}8.5 \pm 0.5 \\
(60)\end{array}$ \\
\hline \multirow[t]{2}{*}{ hRV2 } & WT & $0.04 \pm 0.003$ & $0.44 \pm 0.1$ & $0.46 \pm 0.2$ \\
\hline & G149C & $>10(>250)$ & $>26(>59)$ & $>27(>58)$ \\
\hline \multirow[t]{2}{*}{ PV1_Sabin } & WT & $2.6 \pm 0.04$ & NA & $33 \pm 1$ \\
\hline & I194F & $>10(>3.8)$ & NA & $>54(>1.6)$ \\
\hline \multirow[t]{3}{*}{ EVD68_CU70 } & WT & $1.4 \pm 0.5$ & $0.13 \pm 0.06$ & $2.4 \pm 0.3$ \\
\hline & K167E_M252L & $>10(>7)$ & $2.9 \pm 1.6(22)$ & $>54(>22)$ \\
\hline & A156T_K167E & $>10(>7)$ & $1.4 \pm 0.3(10)$ & $>54(>22)$ \\
\hline
\end{tabular}

\subsection{Sequencing}

Viral RNA was isolated from virus stocks with Nucleospin RNA virus (Macherey-Nagel) according to manufacturer protocol. CDNA fragments were amplified with strain-specific primers using the one step RT-PCR kit (Qiagen). Sequencing was performed by Macrogen Europe. VectorNTI and SnapGene software were used for analysis. et al., 2014b), pT7/EV-A71BrCr and pCMV/hRV14 by using the QuikChange II XL Site-Directed Mutagenesis Kit (Agilent Technologies). All mutants were verified by sequencing after mutagenesis. RNA was obtained by using a T7 RiboMAX Large Scale RNA Production System (Promega) and infectious viruses were generated by transfecting RNA into respective cells with the TransIT-mRNA Transfection Kit (Mirus). pCMV/hRV14 plasmid was transfected with TransIT-LT1 Transfection Reagent (Mirus) into HeLa cells.

\subsection{Molecular modelling}

A Molecular dynamics simulation with explicit water molecules was performed using the Amber18 software (D.A. Case, I.Y. Ben-Shalom, S.R. Brozell, D.S. Cerutti, T.E. Cheatham, III, V.W.D. Cruzeiro, T.A. Darden, R.E. Duke, D. Ghoreishi, M.K. Gilson, H. Gohlke, A.W. Goetz, D. Greene, R Harris, N. Homeyer, S. Izadi, A. Kovalenko, T. Kurtzman, T.S. Lee, S. LeGra, 2018) for the WT pentamer (PDB entry 3vdd (Feil et al., 2012)) and a pentamer where all five g149.a were mutated to cysteine. 4 simulations were setup starting from the pentamer structure in pdb entry 3vdd: a wt pentamer with and without 5 vapendavir molecules bound; the g149c.a pentamer with and without 5 vapendavir inhibitors. A total of $60 \mathrm{~ns}$ was simulated for all systems. We obtained stable simulations for all systems. Details of the simulation parameters are given in Table S2. The LIGPLOT program was used to inspect the interactions in pentamer-vapendavir complexes (Wallace et al., 1995). Visualization of structures was performed with UCSF Chimera (Pettersen et al., 2004).

\subsection{Site-directed mutagenesis}

Mutagenesis was performed on the plasmids pT7/S1F PV1 (Tijsma

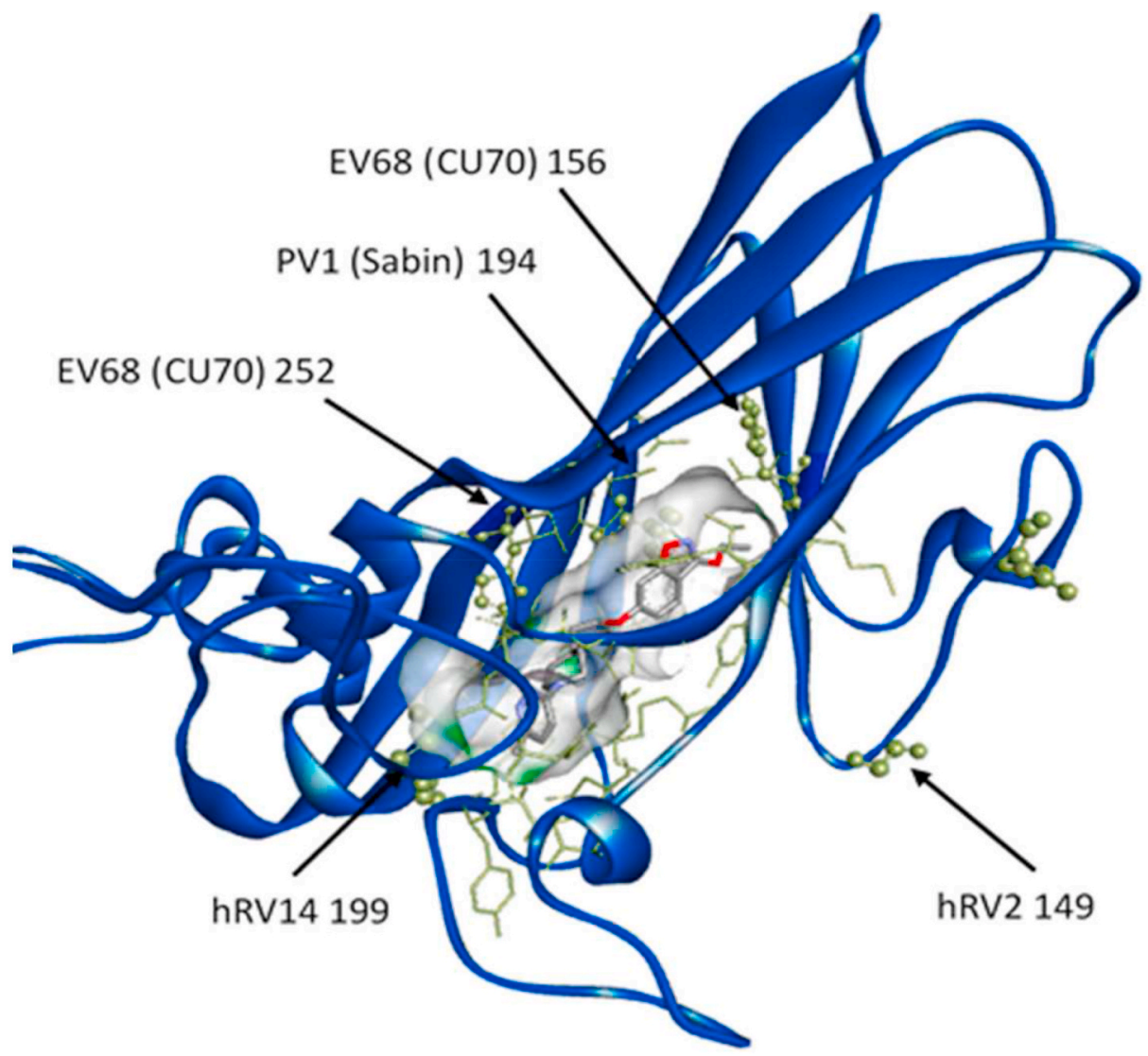

Fig. 1. Location of identified mutated residues corresponding to the residues in hRV2 VP1 protein in complex with Vapendavir. VP1 protein in blue. Pocket surface in grey with drug-interacting residues in yellow. Residues mutated in resistant populations represented in sticks and balls. 
A

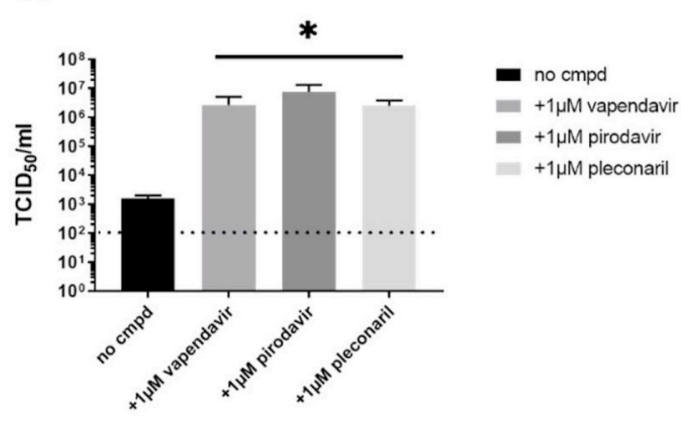

C

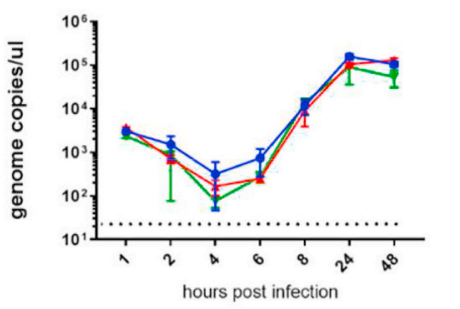

$\rightarrow$ WT

$+\mathrm{C} 3$

* C3+1нM Vapendavir

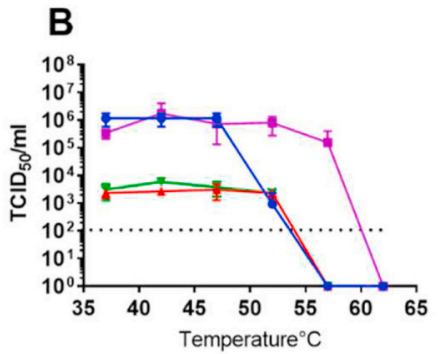

$\rightarrow$ WT

- WT $+5 \mu \mathrm{M}$ Vapendavir

$-\mathrm{c} 3$

$\rightarrow$ C $3+5 \mu \mathrm{M}$ Vapendavir
D

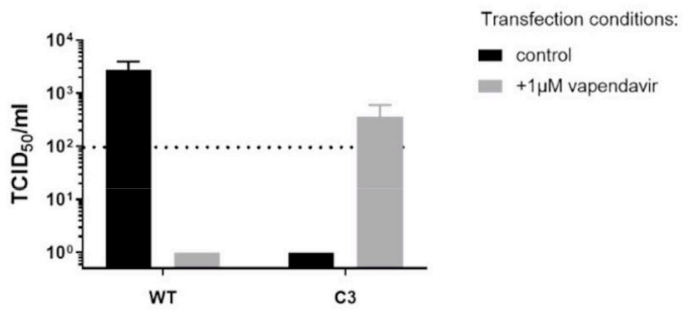

E

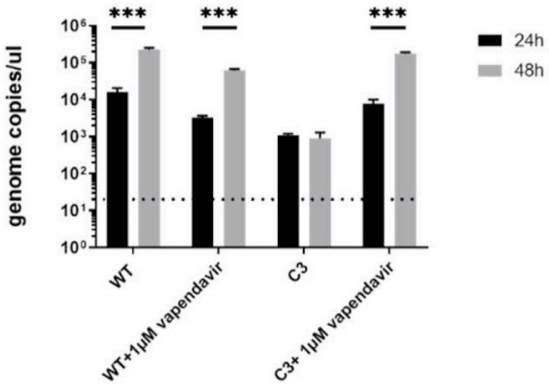

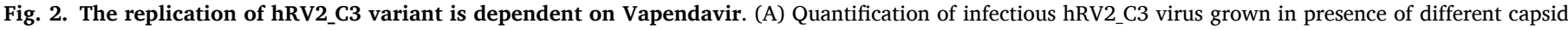

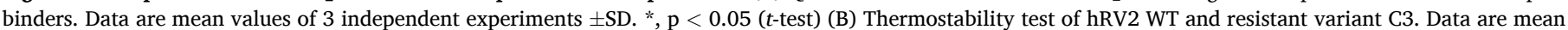

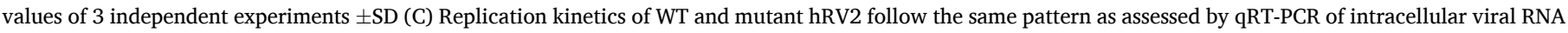

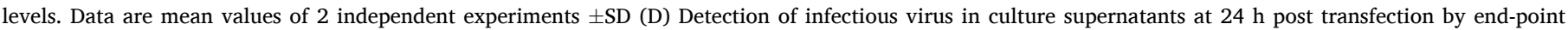

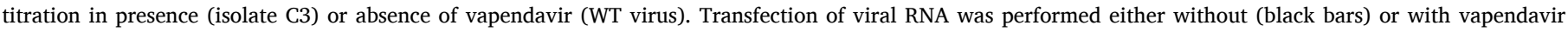

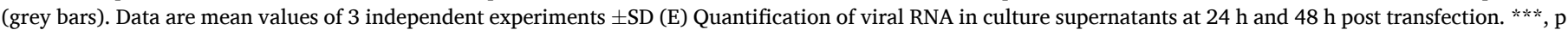
$<0.0005$, analyzed by $t$-test. Data are mean values of 3 independent experiments \pm SD.

\section{Results}

\subsection{Vapendavir-resistant variants are readily selected in cell culture}

To obtain vapendavir-resistant isolates of hRV14, hRV2, EV-D68 (CU70) and PV1 (Sabin) a 5-step resistance selection protocol was employed (Delang et al., 2014). For each virus, the optimal resistance selection conditions were determined and three 96-well plates were set up for selection. We were able to obtain up to 7 potentially resistant populations for each virus. Virus stocks of these isolates were generated in further selection steps and the activity of vapendavir against these isolates was assessed in a multicycle CPE-reduction assay. Seven (7) resistant isolates of hRV14, 3 of PV1_Sabin, 1 of hRV2, and 2 of EV-D68 (CU70) were obtained. All the isolates were fully resistant against vapendavir (Table 1).

\subsection{Mutations in VP1 confer the capsid binder-resistant phenotype}

We next sequenced the capsid region of the resistant isolates and identified amino acid substitutions in the VP1 protein as expected (Table 1). The resistant variants of hRV2, hRV14 and PV1 (Sabin) carried single amino acid substitutions in the VP1 region, whereas EV-D68 (CU70) variants carried double mutations with K167E present in both isolates. Reverse-engineering of the mutations identified in hRV14 and PV1 (Sabin) was performed and revealed that the introduction of respective mutations into the WT backbone resulted in a resistant phenotype (Table S1). To study whether the vapendavir-resistant isolates are cross-resistant against other capsid binders, the activity of two other capsid binders, pleconaril and pirodavir, against the vapendavirresistant isolates was assessed. Cross-resistance to both compounds was observed for all isolates with $>10$ fold reduction in activity 


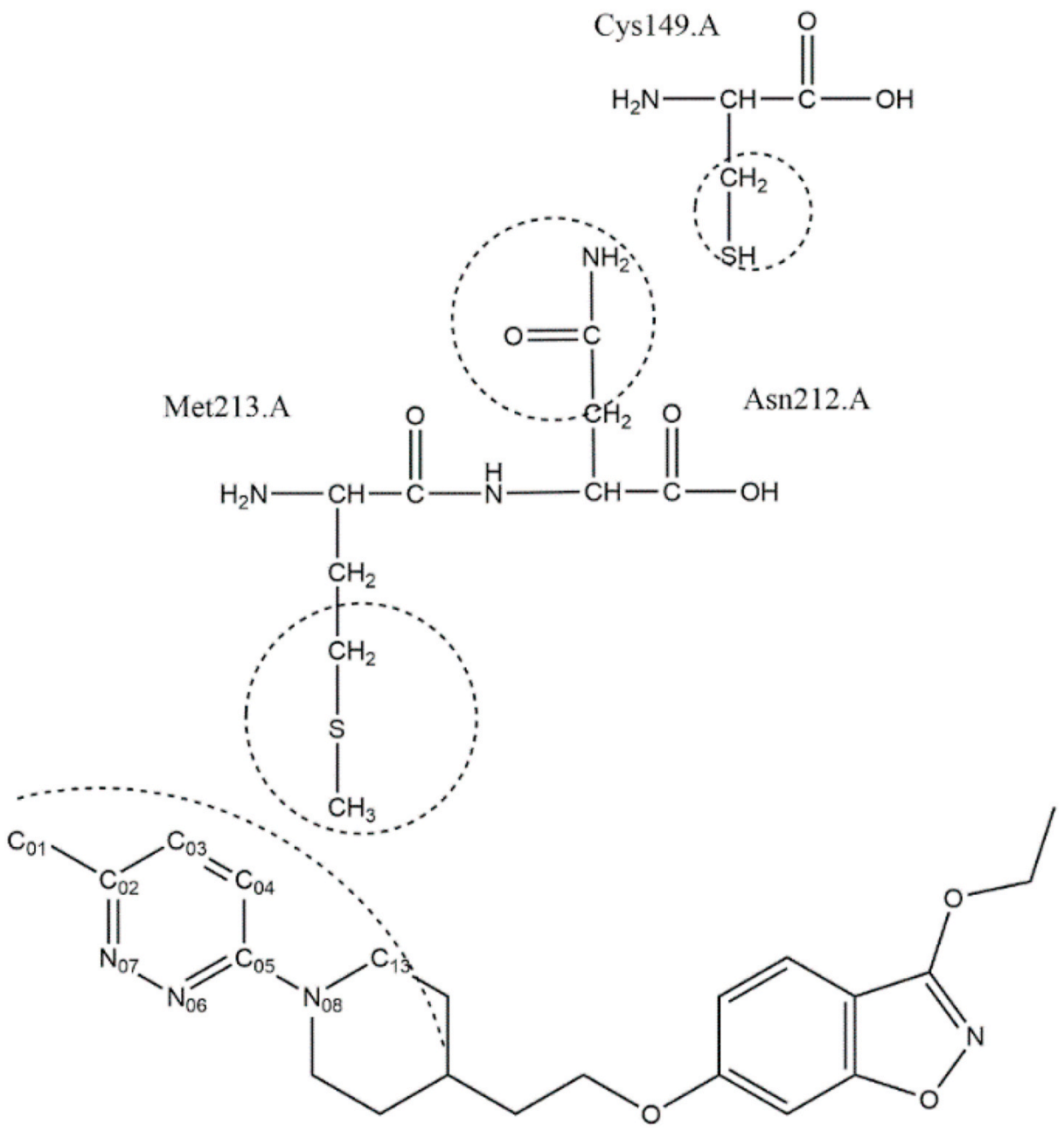

Fig. 3. Vapendavir interaction map with VP1 residues of hRV2.

(Table 1).

\subsection{Location of mutations in VP1 structure}

To determine the location of the identified substitutions in VP1, we superimposed available VP1 crystal structures of the virus strains used in our study and marked the location of mutated residues on the available (PDB: 3vdd) structure of hRV2 in complex with vapendavir (Fig. 1, Fig. S1) (Feil et al., 2012). The substitutions in the resistant populations of hRV14 and PV1 (Sabin) are located in the well-known drug-binding pocket (Heinz et al., 1989; Tijsma et al., 2014b), whereas the substitution in hRV2 is located outside the pocket in one of the VP1 loops not directly interacting with the compound. The K167E residue identified in both isolates of EV-D68 is located on the same loop outside the drug-binding pocket as in hRV2; however, two other residues (A156T and M252L) observed in EV-D68 resistant isolates are present in the drug-binding pocket.

\subsection{The infectivity of the hRV2 vapendavir-resistant isolate is dependent on vapendavir}

The vapendavir-resistant hRV2-G149C isolate, referred here as isolate C3 exhibited a unique phenotype, i.e. infectious virus titers were much lower for this mutant compared to WT hRV2, which was not the case for the resistant isolates of hRV14 and EV-D68 (Fig. S2). Much higher infectious titers were obtained when hRV2_C3 was titrated in the presence of $1 \mu \mathrm{g} / \mathrm{mL}$ of either vapendavir, pleconaril or pirodavir
(Fig. 2A). The hRV2_C3 populations from the cultures that exhibited CPE at the highest dilution of the virus stock (1/100 without compound and $1 / 1000$ in presence of vapendavir) were sequenced. Intriguingly, the virus cultured in the absence of the drug lost the G149C mutation.

Next viral RNA of the C3 mutant and WT were transfected in cells to test whether infectious virions can be produced in presence or absence of vapendavir (Fig. 2D). No infectious C3 virus was detectable in the culture supernatant at $24 \mathrm{~h}$ and $48 \mathrm{~h}$ post transfection in the absence of antiviral pressure. However, when transfection of cultures with C3 RNA was performed in medium that was supplemented with $1 \mu \mathrm{M}$ of vapendavir, infectious virus was obtained and could be detected by endpoint titration in vapendavir-containing medium. This was not the case in the absence of the compound. Thus, the infectivity of C3 depends on vapendavir. There was also no increase in viral RNA copies from $24 \mathrm{~h}$ to $48 \mathrm{~h}$ in C3 transfected cultures in absence of vapendavir. In contrast, when $1 \mu \mathrm{M}$ of vapendavir was added to the transfected culture, $1 \log _{10}$ increase in viral genome copies from $24 \mathrm{~h}$ to $48 \mathrm{~h}$ was detected as compared to untreated control, suggesting that vapendavir is essential for the development of replication-competent particles (Fig. 2E).

\subsection{Vapendavir does not protect the hRV2_C3 resistant mutant against heat-inactivation}

The binding of WIN-like compounds to the viral capsid is known to make the virus less sensitive to heat-inactivation (Groarke and Pevear, 1999; Rombaut et al., 1991). It has been previously reported that the mutations in the drug-binding pocket abolish the interaction between 


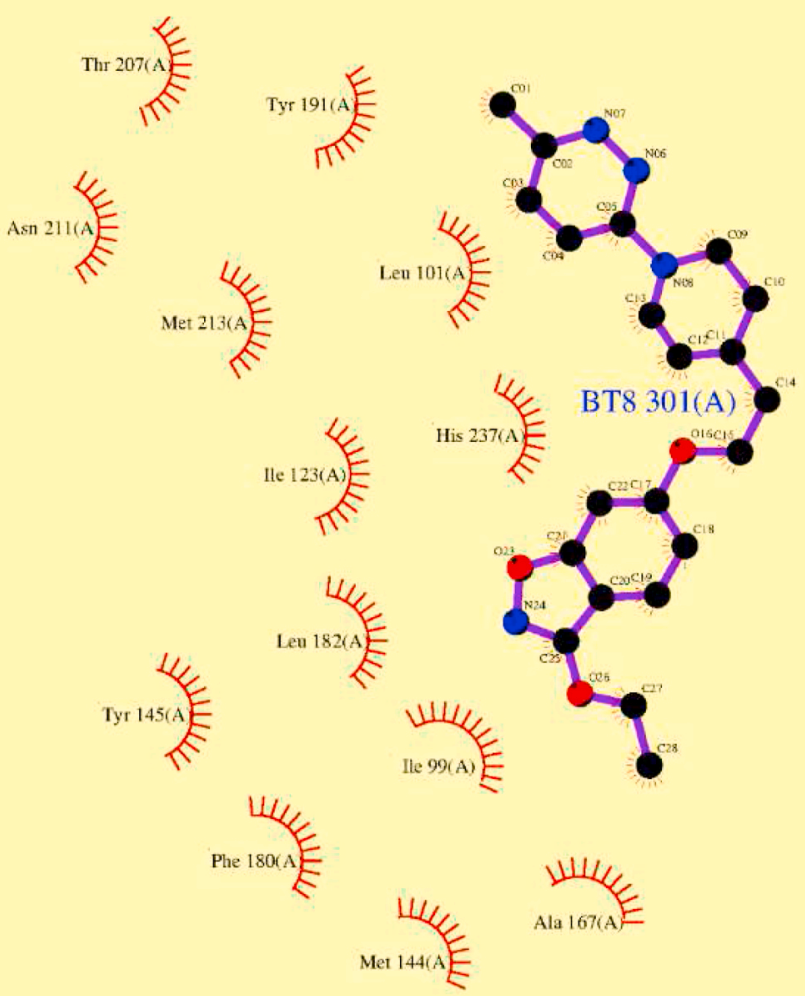

Key

Ligand bond
Non-ligand bond
Hydrogen bond and its length

His 53 Non-ligand residues involved in hydrophobic

भim contact(s)

- Corresponding atoms involved in hydrophobic contact(s)

\section{$3 v d d$}

Fig. 4. Ligplot map of interactions of the HRV2 capsid with vapendavir in pdb entry 3vdd. M213 makes van der Waals contact with the inhibitor.

the capsid and the compound and as a consequence no protection against heat-inactivation is observed. To explore whether this type of interaction is possible with hRV2_C3, we performed a thermostability test (Fig. 2B). The WT virus was inactivated at $56{ }^{\circ} \mathrm{C}$ in the absence of compound and in presence of $5 \mu \mathrm{M}$ of vapendavir at $62^{\circ} \mathrm{C}$. This shift was not observed with the resistant isolate, indicating that vapendavir has no influence on heat-inactivation of the virus. In addition, there was no decrease in infectivity of the resistant isolate at $52{ }^{\circ} \mathrm{C}$ as was observed for the WT virus. HRV2_C3 has however, the same plaque size as the WT (data not shown), also the replication kinetics of the isolate and WT virus are comparable (Fig. 2C).

\subsection{The conserved glycine at VP1 position 149 is important for enterovirus infectivity}

Since the G149 residue is conserved in many enterovirus species (Fig. S3), we introduced the G to C (G156C in RV14, G159C in EV-A71) substitution in infectious clones of hRV14 and EV-A71 BrCr and determined whether this would result in a similar resistant/dependent phenotype. Following transfection of the mutated hRV14 or EV-A71 infectious clone in HeLa cells, no infectious virus was detectable either in the presence or in absence of vapendavir. However, when the supernatant from the cultures transfected with EV-A71 G149C was passaged in absence of vapendavir, the virus reverted to WT and CPE was observed. No resistance or dependency on vapendavir was thus observed with the G to C substitution in EV-A71 or hRV14 background.

\subsection{Molecular modelling of the resistance mechanism of hRV2 isolate to vapendavir}

Molecular modelling was used to explore whether the G149C in hRV2 affects the inhibitor binding in the pocket via network interactions (Fig. 3). The ligplot analysis (Wallace et al., 1995) of 3vdd.pdb depicting the inhibitor interactions with surrounding amino acids (Fig. 4) reveals that M213 is one of the amino acids that may stabilize the binding of vapendavir by van der Waals (vdw) interactions. A contact analysis from the MD trajectory of the unliganded mutant simulation shows that there are many (vdw) contacts between the sidechains of C149 and N212 (Table S3). This is also clear from a representative structure from the MD simulation of the mutant with vapendavir (Fig. 5). Next, the Root mean square deviations (RMSD) and fluctuations (RMSF) for the 2 loops of amino acids 210-214 (containing M213, green ribbon in Fig. 5) and 


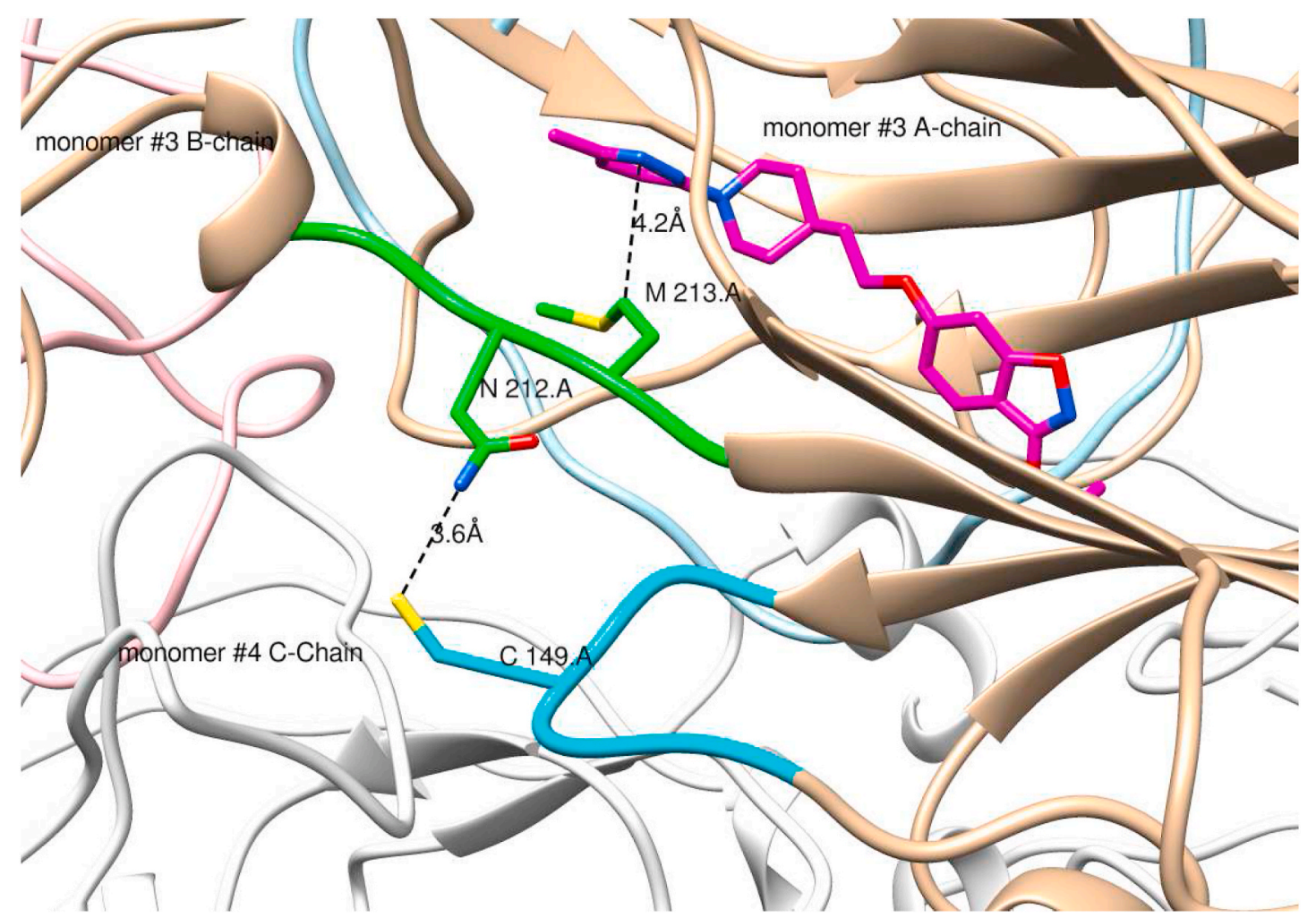

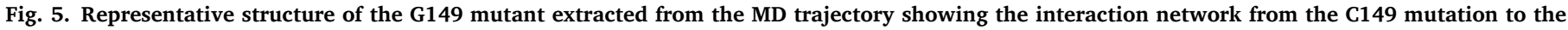

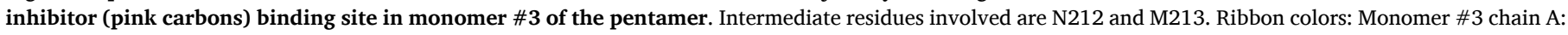

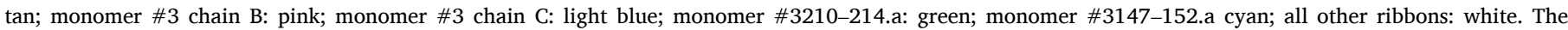

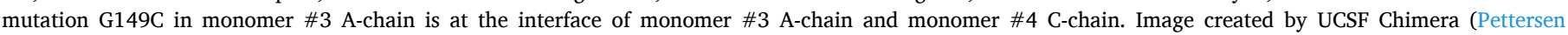
et al., 2004).

amino acids 147-151 (containing the G to C149 mutation, cyan ribbon in Fig. 5) in both WT and mutant (liganded and unliganded) simulations were explored. However, no significant differences between wildtype and mutant were observed.

\section{Discussion}

We here studied the resistance profile to vapendavir of different enteroviruses. In line with earlier reports, resistant mutants were readily selected in cell culture for all 4 viruses used in the study. The isolates all carried the mutations in the VP1 protein, where the known binding pocket for capsid binders is located. Furthermore, all the isolates proved resistant to other capsid binders confirming the same mechanism of action. Interestingly, we identified two types of mutations conferring the resistant phenotype: i.e. in amino acids lining the binding pocket but also amino acids outside the pocket. Mutants outside the pocket have also been reported in hRV14 isolates resistant to WIN 52084 (Heinz et al., 1989). However, the mutations identified in hRV2 and EV-D68 are localized in a different loop of the VP1 protein outside the pocket than reported in that study.

We identified pocket mutations in PV1 (Sabin), EV-D68 and hRV14. The pocket mutations impair the binding of WIN-like compounds and thus abolish their antiviral activity. The I194F mutation in poliovirus, as identified here, has been shown to confer resistance to other capsid binders, i.e. H1PVAT and V-073 (Liu et al., 2012; Tijsma et al., 2014b). According to modelling studies, the I194 amino acid is important for the interaction of the capsid binder with the pocket and the mutation in this position can have an effect on compound binding (Tijsma et al., 2014b). The pocket mutation at position $\mathrm{C} 199$ (located at the pocket entrance) in hRV14 was identified previously in a pleconaril-resistant isolate (Feil et al., 2012) and in WIN 52084-resistant isolates (Heinz et al., 1989). A mutation at the entrance to the pocket has been reported as well in a CVB3 isolate resistant to pirodavir (Braun et al., 2015). In that case, molecular modelling suggested that mutations in residue I1207 to K or R are preventing the compound from entering the canyon.

Previously reported pleconaril-resistant hRV2 isolates carry the I99F mutation located in the binding pocket (Feil et al., 2012). The EV68 mutation V69A in a pleconaril-resistant variant is also located in the pocket (Sun et al., 2015). The pocket mutation A156T identified here in vapendavir-resistant isolate of EV-D68 corresponds to the A150 T/V mutation reported in hRV14 pleconaril-resistant (Feil et al., 2012). However, we here observed mutations in hRV2 and EV-D68 that are located outside the drug binding pocket. Interestingly, the hRV2 resistant isolate had a particular vapendavir-dependent phenotype; its infectivity is dependent on the presence of vapendavir and its replication is impaired in the absence of the drug. On the other hand, the resistant EV-D68 variants carrying the K167E mutation in the same loop of VP1 protein could replicate independently of vapendavir, and their phenotype was similar to that of the isolates with the mutations inside the binding pocket. It is likely that the mutations in the pocket A156T and M252L are primary responsible for the resistant phenotype of EV-D68, since the glutamic acid at the same position as K167E in CU70 strain (K155 in the prototype Fermon strain) is naturally present in several EV-D68 strains, that are inhibited by capsid binders (e.g. strain 4311000742, 4310900947 and 4310902042). Moreover, this amino acid is present in the 4310901348 strain, which is susceptible to pleconaril and pirodavir, but not vapendavir inhibition (Sun et al., 2015). This suggests that this particular residue is not crucial for the antiviral activity of capsid binders. The K167E amino acid change was reported as a secondary mutation in one of the pleconaril-resistant EV-D68 isolates (Sun et al., 2015). Taken together, the K167E substitution observed here in the EV-D68 resistant isolates could be beneficial for virus fitness, but 
is unlikely to underlie the resistant phenotype.

\subsection{Dependency of $h R V 2_{-}$C3 variant on vapendavir}

The dependency of picornavirus replication on antivirals has been reported before. For example a $\mathrm{Gua}-\mathrm{HCl}$ dependent PV1 virus with mutations in $2 \mathrm{C}$ protein can only replicate in the presence of Gua-HCl (Pincus et al., 1986). The dependence of echovirus on rhodanin is attributed to the F53Y mutation in the structural protein VP4 (Kraus et al., 1997). Dependency of PV3 (Sabin) on WIN 51711 was attributed to mutations in the inside lining of the capsid (Mosser et al., 1994; Mosser and Rueckert, 1993).

The hRV2_C3 mutant virus identified here is dependent on the inhibitor for efficient dissemination. Since the isolate has the same replication kinetics as the WT virus and is able to release viral progeny (as shown by detection of the viral RNA in the supernatant of transfected cultures), we suggest that vapendavir binding may be required for the proper conformation of the mutant virus particle allowing it to efficiently bind/enter the cells.

The lack of thermostabilizing effect of vapendavir in the mutant hRV2 suggests that the binding of the compound to the mutant virus differs from the binding to the WT virus. On the other hand, the mutant virus still retains its infectivity at $52{ }^{\circ} \mathrm{C}$, whereas the WT variant does not. Since hRV2_C3 stocks were prepared in the presence of the compound it cannot be excluded that the stability of the mutant isolate at 52 ${ }^{\circ} \mathrm{C}$ is due to some remaining vapendavir (carry-over effect). Extreme thermolability of WIN 51711-dependent poliovirus mutant particles has been reported (Mosser et al., 1994; Mosser and Rueckert, 1993). WIN_51711 was shown to stabilize the infectious particles without affecting their infectivity. However, the level of resistance and replication efficiency reported in that study is apparently less pronounced than with our hRV2_C3 isolate. Since G149C variant was more stable at increased temperatures, the phenotype cannot be explained by thermolability as in the case of WIN_51711-dependent poliovirus.

Our molecular simulation data do not reveal an impairment of vapendavir binding to the mutated VP1 protein of hRV2. This can support the hypothesis that the compound is still capable of binding and stabilizing the mutant hRV2 virus. However, it is possible that the effect of the mutation in simulations may only appear after much longer simulation times. Passaging of hRV2_C3 or EV-A71 BrCr (G159C) without antiviral pressure results in a rapid reverting to wild-type, indicating that the mutation has an infavourable effect on the virus. Based on the data from our experiments, the genome replication and release of viral particles is unaffected by G149C substitution, however these particles are not able to infect the cells without the presence of vapendavir. We therefore speculate that $\mathrm{G} 149 \mathrm{C}$ might exert its effects during dynamic processes of viral life cycle, when the capsid is subject to conformational changes such as particle assembly or attachment/ uncoating. G149C particles might require vapendavir for either stability after release or for keeping proper conformation of VP1 for efficient entry into the host cell. Further structural studies might provide detailed explanation of our observations.

In conclusion, vapendavir results, depending on the virus that it inhibits, in various drug-resistant geno- and phenotypes. Like other capsid binders it results rapidly in the selection of drug-resistant variants (Benschop et al., 2015; Collett et al., 2016; Pevear et al., 2005). The combination of capsid binders with other entero-/rhinovirus inhibitors that have a different mechanism of antiviral activity (GPEI) may be worth considering.

\section{Transparency declarations}

All other authors: none to declare. Vapendavir is the property of Vaxart Inc.

\section{Declaration of competing interest}

The authors declare that they have no known competing financial interests or personal relationships that could have appeared to influence the work reported in this paper.

\section{Acknowledgements}

We would like to thank Vaxart Inc, Dr. Vernachio and Dr. Tucker for providing vapendavir for this study. This project has received funding from the European Union's Horizon 2020 research and innovation programme under the Marie Sklodowska-Curie grant agreement No 642434. L.S. was funded by the China Scholarship Council (CSC) grant 201403250056.

\section{Appendix A. Supplementary data}

Supplementary data to this article can be found online at https://doi. org/10.1016/j.antiviral.2021.105177.

\section{References}

Abzug, M.J., Cloud, G., Bradley, J., Sánchez, P.J., Romero, J., Powell, D., Lepow, M., Mani, C., Capparelli, E.V., Blount, S., Lakeman, F., Whitley, R.J., Kimberlin, D.W., 2003. Double blind placebo-controlled trial of pleconaril in infants with enterovirus meningitis. Pediatr. Infect. Dis. J. 22, 335-340. https://doi.org/10.1097/00006454200304000-00009.

Abzug, M.J., Michaels, M.G., Wald, E., Jacobs, R.F., Romero, J.R., Sánchez, P.J., Wilson, G., Krogstad, P., Storch, G.A., Lawrence, R., Shelton, M., Palmer, A., Robinson, J., Dennehy, P., Sood, S.K., Cloud, G., Jester, P., Acosta, E.P., Whitley, R., Kimberlin, D., National Institute of Allergy and Infectious Diseases Collaborative Antiviral Study Group, 2015. A randomized, double-blind, placebo-controlled trial of pleconaril for the treatment of neonates with enterovirus sepsis. J. Pediatric Infect. Dis. Soc. 5, piv015. https://doi.org/10.1093/jpids/piv015.

Aliabadi, N., Messacar, K., Pastula, D.M., Robinson, C.C., Leshem, E., Sejvar, J.J., Nix, W. A., Oberste, M.S., Feikin, D.R., Dominguez, S.R., 2016. Enterovirus D68 infection in children with acute flaccid myelitis, Colorado, USA, 2014. Emerg. Infect. Dis. 22, 1387-1394. https://doi.org/10.3201/eid2208.151949.

Baggen, J., Thibaut, H.J., Strating, J.R.P.M., Van Kuppeveld, F.J.M., 2018. The life cycle of non-polio enteroviruses and how to target it. Nat. Rev. Microbiol. https://doi.org/ 10.1038/s41579-018-0005-4.

Benschop, K.S.M., Wildenbeest, J.G., Koen, G., Minnaar, R.P., van Hemert, F.J., Westerhuis, B.M., Pajkrt, D., van den Broek, P.J., Vossen, A.C.T.M., Wolthers, K.C., 2015. Genetic and antigenic structural characterization for resistance of echovirus 11 to pleconaril in an immunocompromised patient. J. Gen. Virol. 96, 571-579. https://doi.org/10.1099/vir.0.069773-0.

Braun, H., Kirchmair, J., Williamson, M.J., Makarov, V.A., Riabova, O.B., Glen, R.C., Sauerbrei, A., Schmidtke, M., 2015. Molecular mechanism of a specific capsid binder resistance caused by mutations outside the binding pocket. Antivir. Res. 123, 138-145. https://doi.org/10.1016/j.antiviral.2015.09.009.

Collett, M.S., Hincks, J.R., Benschop, K., Duizer, E., van der Avoort, H., Rhoden, E., Liu, H., Oberste, M.S., McKinlay, M.A., Hartford, M., 2016. Antiviral activity of pocapavir in a randomized, blinded, placebo-controlled human oral poliovirus vaccine challenge model. J. Infect. Dis. 215, jiw542. https://doi.org/10.1093/ infdis/jiw542.

Case, D.A., Ben-Shalom, I.Y., Brozell, S.R., Cerutti, D.S., Cheatham, T.E., Cruzeiro III, V. W.D., Darden, T.A., Duke, R.E., Ghoreishi, D., Gilson, M.K., Gohlke, H., Goetz, A.W., Greene, D., Harris, R., Homeyer, N., Izadi, S., Kovalenko, A., Kurtzman, T., Lee, T.S., LeGra, S., 2018. AMBER 2018. D.M.Y. and P.A.K.

Delang, L., Segura Guerrero, N., Tas, A., Quérat, G., Pastorino, B., Froeyen, M., Dallmeier, K., Jochmans, D., Herdewijn, P., Bello, F., Snijder, E.J., de Lamballerie, X., Martina, B., Neyts, J., van Hemert, M.J., Leyssen, P., 2014. Mutations in the chikungunya virus non-structural proteins cause resistance to favipiravir (T-705), a broad-spectrum antiviral. J. Antimicrob. Chemother. 69, 2770-2784. https://doi.org/10.1093/jac/dku209.

Diana, G.D., Salvador, U.J., Zalay, E.S., Johnson, R.E., Collins, J.C., Johnson, D., Hinshaw, W.B., Lorenz, R.R., Thielking, W.H., Pancic, F., 1977. Antiviral activity of some $\beta$-diketones. 1 . Aryl alkyl diketones. In vitro activity against both RNA and DNA viruses. J. Med. Chem. 20, 750-756. https://doi.org/10.1021/jm00216a003.

Feil, S.C., Hamilton, S., Krippner, G.Y., Lin, B., Luttick, A., McConnell, D.B., Nearn, R., Parker, M.W., Ryan, J., Stanislawski, P.C., Tucker, S.P., Watson, K.G., Morton, C.J., 2012. An orally available 3-ethoxybenzisoxazole capsid binder with clinical activity against human rhinovirus. ACS Med. Chem. Lett. 3, 303-307. https://doi.org/ $10.1021 / \mathrm{ml} 2002955$.

Gern, J.E., 2015. How rhinovirus infections cause exacerbations of asthma. Clin. Exp. Allergy 45, 32-42. https://doi.org/10.1111/cea.12428.

Groarke, J.M., Pevear, D.C., 1999. Attenuated virulence of pleconaril-resistant coxsackievirus B3 variants. J. Infect. Dis. 179, 1538-1541. https://doi.org/10.1086/ 314758. 
Hayden, F.G., Herrington, D.T., Coats, T.L., Kim, K., Cooper, E.C., Villano, S.A., Liu, S., Hudson, S., Pevear, D.C., Collett, M., McKinlay, M., 2003. Efficacy and safety of oral pleconaril for treatment of colds due to picornaviruses in adults: results of 2 doubleblind, randomized, placebo-controlled trials. Clin. Infect. Dis. 36, 1523-1532. https://doi.org/10.1086/375069.

Hayden, F.G., Hipskind, G.J., Woerner, D.H., Eisen, G.F., Janssens, M., Janssen, P.A., Andries, K., 1995. Intranasal pirodavir $(\mathrm{R} 77,975)$ treatment of rhinovirus colds. Antimicrob. Agents Chemother. 39, 290-294.

Heinz, B.A., Rueckert, R.R., Shepard, D.A., Dutko, F.J., McKinlay, M.A., Fancher, M., Rossmann, M.G., Badger, J., Smith, T.J., 1989. Genetic and molecular analyses of spontaneous mutants of human rhinovirus 14 that are resistant to an antiviral compound. J. Virol. 63, 2476-2485.

Imamura, T., Oshitani, H., 2015. Global reemergence of enterovirus D68 as an important pathogen for acute respiratory infections. Rev. Med. Virol. 25, 102-114. https://doi. org/10.1002/rmv.1820.

Kirkpatrick, B.D., Mychaleckyj, J.C., 2015. Polio eradication: inching forward, with safety nets. Lancet Infect. Dis. 15, 1244-1245. https://doi.org/10.1016/S1473-3099 (15)00261-3.

Kraus, W., Zimmermann, H., Eggers, H.J., Nelsen-salz, B., 1997. Rhodanine Resistance and Dependence of Echovirus 12 : a Possible Consequence of Capsid Flexibility, vol 71, pp. 1697-1702.

Lacroix, C., Querol-Audí, J., Roche, M., Franco, D., Froeyen, M., Guerra, P., Terme, T., Vanelle, P., Verdaguer, N., Neyts, J., Leyssen, P., 2014. A novel benzonitrile analogue inhibits rhinovirus replication. J. Antimicrob. Chemother. 69, 2723-2732. https://doi.org/10.1093/jac/dku200.

Liang, Z., Wang, J., 2014. EV71 vaccine, an invaluable gift for children. Clin. Transl. Immunol. 3, e28. https://doi.org/10.1038/cti.2014.24.

Liu, H.-M., Roberts, J.A., Moore, D., Anderson, B., Pallansch, M.A., Pevear, D.C., Collett, M.S., Oberste, M.S., 2012. Characterization of poliovirus variants selected for resistance to the antiviral compound V-073. Antimicrob. Agents Chemother. 56, 5568-5574. https://doi.org/10.1128/AAC.00539-12.

Lönnrot, M., Sjöroos, M., Salminen, K., Maaronen, M., Hyypiä, T., Hyöty, H., 1999. Diagnosis of enterovirus and rhinovirus infections by RT-PCR and time-resolved fluorometry with lanthanide chelate labeled probes. J. Med. Virol. 59, 378-384.

Mallia, P., Message, S.D., Gielen, V., Contoli, M., Gray, K., Kebadze, T., Aniscenko, J., Laza-Stanca, V., Edwards, M.R., Slater, L., Papi, A., Stanciu, L.A., Kon, O.M., Johnson, M., Johnston, S.L., 2011. Experimental rhinovirus infection as a human model of chronic obstructive pulmonary disease exacerbation. Am. J. Respir. Crit. Care Med. 183, 734-742. https://doi.org/10.1164/rccm.201006-08330C.

Matz, J., 2013. Vapendavir significantly improves upper respiratory symptoms of naturally acquired rhinovirus infection in asthmatic adults: results of a phase 2 clinical trial. Eur. Respir. J. 42.

McGoldrick, A., Macadam, A.J., Dunn, G., Rowe, A., Burlison, J., Minor, P.D., Meredith, J., Evans, D.J., Almond, J.W., 1995. Role of mutations G-480 and C-6203 in the attenuation phenotype of Sabin type 1 poliovirus. J. Virol. 69, 7601-7605.

McKinlay, M.A., Miralles, J.V., Brisson, C.J., Pancic, F., 1982. Prevention of human poliovirus-induced paralysis and death in mice by the novel antiviral agent arildone. Antimicrob. Agents Chemother. 22, 1022-1025. https://doi.org/10.1128/ aac.22.6.1022.

Messacar, K., Asturias, E.J., Hixon, A.M., Van Leer-Buter, C., Niesters, H.G.M., Tyler, K. L., Abzug, M.J., Dominguez, S.R., 2018. Enterovirus D68 and acute flaccid myelitis - evaluating the evidence for causality. Lancet Infect. Dis. 18, e239-e247. https://doi.org/10.1016/S1473-3099(18)30094-X.

Messacar, K., Schreiner, T.L., Van Haren, K., Yang, M., Glaser, C.A., Tyler, K.L., Dominguez, S.R., 2016. Acute flaccid myelitis: a clinical review of US cases 2012 2015. Ann. Neurol. 80, 326-338. https://doi.org/10.1002/ana.24730.
Mishra, N., Ng, T.F.F., Marine, R.L., Jain, K., Ng, J., Thakkar, R., Caciula, A., Price, A., Garcia, J.A., Burns, J.C., Thakur, K.T., Hetzler, K.L., Routh, J.A., Konopka-Anstadt, J. L., Nix, W.A., Tokarz, R., Briese, T., Oberste, M.S., Lipkin, W.I., 2019. Antibodies to enteroviruses in cerebrospinal fluid of patients with acute flaccid myelitis. mBio 10, e01903-e01919. https://doi.org/10.1128/MBIO.01903-19.

Mosser, A.G., Rueckert, R.R., 1993. WIN 51711-dependent mutants of poliovirus type 3: evidence that virions decay after release from cells unless drug is present. J. Virol. 67, 1246-1254.

Mosser, A.G., Sgro, J.Y., Rueckert, R.R., 1994. Distribution of drug resistance mutations in type 3 poliovirus identifies three regions involved in uncoating functions. J. Virol. 68, 8193-8201.

Passioti, M., Maggina, P., Megremis, S., Papadopoulos, N.G., 2014. The common cold: potential for future prevention or cure topical collection on rhinosinusitis. Curr. Allergy Asthma Rep. 14, 413. https://doi.org/10.1007/s11882-013-0413-5.

Pettersen, E.F., Goddard, T.D., Huang, C.C., Couch, G.S., Greenblatt, D.M., Meng, E.C., Ferrin, T.E., 2004. UCSF Chimera - a visualization system for exploratory research and analysis. J. Comput. Chem. 25, 1605-1612. https://doi.org/10.1002/jcc. 20084.

Pevear, D.C., Hayden, F.G., Demenczuk, T.M., Barone, L.R., McKinlay, M.A., Collett, M. S., 2005. Relationship of pleconaril susceptibility and clinical outcomes in treatment of common colds caused by rhinoviruses. Antimicrob. Agents Chemother. 49, 4492. https://doi.org/10.1128/AAC.49.11.4492-4499.2005.

Pincus, S.E., Diamond, D.C., Emini, E.A., Wimmer, E., 1986. Guanidine-selected mutants of poliovirus: mapping of point mutations to polypeptide 2C. J. Virol. 57, 638.

Polio Now - GPEI [WWW Document], n.d. URL http://polioeradication.org/poli o-today/polio-now/(accessed 4.2.19)..

Rhoden, E., Liu, H.-M., Wang-Chern, S., Oberste, M.S., 2013. Anti-poliovirus activity of protease inhibitor AG-7404, and assessment of in vitro activity in combination with antiviral capsid inhibitor compounds. Antivir. Res. 98, 186-191. https://doi.org/ 10.1016/j.antiviral.2013.03.003.

Rombaut, B., Andries, K., Boeye, A., 1991. A comparison of WIN 51711 and R 78206 as stabilizers of poliovirus virions and procapsids. J. Gen. Virol. 72, 2153-2157. https://doi.org/10.1099/0022-1317-72-9-2153.

Shepard, D.A., Heinz, B.A., Rueckert, R.R., 1993. WIN 52035-2 inhibits both attachment and eclipse of human rhinovirus 14. J. Virol. 67, 2245-2254.

Sun, L., Meijer, A., Froeyen, M., Zhang, L., Thibaut, H.J., Baggen, J., George, S., Vernachio, J., van Kuppeveld, F.J.M., Leyssen, P., Hilgenfeld, R., Neyts, J., Delang, L., 2015. Antiviral activity of broad-spectrum and enterovirus-specific inhibitors against clinical isolates of enterovirus D68. Antimicrob. Agents Chemother. 59, 7782-7785. https://doi.org/10.1128/AAC.01375-15.

Tapparel, C., Siegrist, F., Petty, T.J., Kaiser, L., 2013. Picornavirus and enterovirus diversity with associated human diseases. Infect. Genet. Evol. 14, 282-293. https:// doi.org/10.1016/J.MEEGID.2012.10.016.

Tijsma, A., Franco, D., Tucker, S., Hilgenfeld, R., Froeyen, M., Leyssen, P., Neyts, J., 2014a. The capsid binder Vapendavir and the novel protease inhibitor SG85 inhibit enterovirus 71 replication. Antimicrob. Agents Chemother. 58, 6990-6992. https:// doi.org/10.1128/AAC.03328-14.

Tijsma, A., Thibaut, H.J., Spieser, S.A.H., De Palma, A., Koukni, M., Rhoden, E., Oberste, S., Pürstinger, G., Volny-Luraghi, A., Martin, J., Marchand, A., Chaltin, P., Neyts, J., Leyssen, P., 2014b. H1PVAT is a novel and potent early-stage inhibitor of poliovirus replication that targets VP1. Antivir. Res. 110, 1-9. https://doi.org/ 10.1016/j.antiviral.2014.07.003.

Uprety, P., Curtis, D., Elkan, M., Fink, J., Rajagopalan, R., Zhao, C., Bittinger, K., Mitchell, S., Ulloa, E.R., Hopkins, S., Graf, E.H., 2019. Association of enterovirus D68 with acute flaccid myelitis. Emerg. Infect. Dis. 25, 1676-1682. https://doi.org/ 10.3201/eid2509.190468. Philadelphia, Pennsylvania, USA, 2009-2018.

Wallace, A.C., Laskowski, R.A., Thornton, J.M., 1995. LIGPLOT: a program to generate schematic diagrams of protein-ligand interactions. Protein Eng. 8, 127-134. 